\title{
Improved 64-bit Radix-16 Booth Multiplier Based on Partial Product Array Height Reduction
}

\author{
Antelo, Elisardo; Montuschi, Paolo; Nannarelli, Alberto
}

Published in:

IEEE Transactions on Circuits and Systems Part 1: Regular Papers

Link to article, DOI:

10.1109/TCSI.2016.2561518

Publication date:

2016

Document Version

Peer reviewed version

Link back to DTU Orbit

Citation $(A P A)$ :

Antelo, E., Montuschi, P., \& Nannarelli, A. (2016). Improved 64-bit Radix-16 Booth Multiplier Based on Partial Product Array Height Reduction. IEEE Transactions on Circuits and Systems Part 1: Regular Papers, 64(2), 409-418. https://doi.org/10.1109/TCSI.2016.2561518

\section{General rights}

Copyright and moral rights for the publications made accessible in the public portal are retained by the authors and/or other copyright owners and it is a condition of accessing publications that users recognise and abide by the legal requirements associated with these rights.

- Users may download and print one copy of any publication from the public portal for the purpose of private study or research.

- You may not further distribute the material or use it for any profit-making activity or commercial gain

- You may freely distribute the URL identifying the publication in the public portal 


\title{
Improved 64-bit Radix-16 Booth Multiplier Based on Partial Product Array Height Reduction
}

\author{
Elisardo Antelo, Paolo Montuschi, Fellow, IEEE, and Alberto Nannarelli, Senior Member, IEEE
}

\begin{abstract}
In this paper, we describe an optimization for binary radix-16 (modified) Booth recoded multipliers to reduce the maximum height of the partial product columns to $\lceil n / 4\rceil$ for $n=64$-bit unsigned operands. This is in contrast to the conventional maximum height of $[(n+1) / 4\rceil$. Therefore, a reduction of one unit in the maximum height is achieved. This reduction may add flexibility during the design of the pipelined multiplier to meet the design goals, it may allow further optimizations of the partial product array reduction stage in terms of area/delay/power and/or may allow additional addends to be included in the partial product array without increasing the delay. The method can be extended to Booth recoded radix-8 multipliers, signed multipliers, combined signed/unsigned multipliers, and other values of $\boldsymbol{n}$.
\end{abstract}

Index Terms-Binary multipliers, modified Booth recoding, radix-16.

\section{INTRODUCTION}

B INARY multipliers are a widely used building block element in the design of microprocessors and embedded systems, and therefore, they are an important target for implementation optimization [1]-[6]. Current implementations of binary multiplication follow the steps of [7]: 1) recoding of the multiplier in digits in a certain number system; 2) digit multiplication of each digit by the multiplicand, resulting in a certain number of partial products; 3 ) reduction of the partial product array to two operands using multioperand addition techniques; and 4) carry-propagate addition of the two operands to obtain the final result.

The recoding type is a key issue, since it determines the number of partial products. The usual recoding process recodes a binary operand into a signed-digit operand with digits in a minimally redundant digit set [7], [8]. Specifically, for radix-r $\left(r=2^{m}\right)$, the binary operand is composed of nonredundant radix- $r$ digits (by just making groups of $m$ bits), and these are recoded from the set $\{0,1, \ldots, r-1\}$ to the

Manuscript received December 16, 2015; revised March 21, 2016; accepted April 1, 2016. The work of E. Antelo was supported in part by the Ministry of Education and Science of Spain, FEDER funds under Contract TIN 201341129-P, and in part by Xunta de Galicia, GRC2014/008. This paper was recommended by Associate Editor S. Ghosh.

E. Antelo is with the Department of Electrónica e Computación, University of Santiago de Compostela, 15782 Santiago de Compostela, Spain (e-mail: elisardo.antelo@usc.es).

P. Montuschi is with the Department of Control and Computer Engineering, Politecnico di Torino, 10129, Italy (e-mail: paolo.montuschi@ polito.it).

A. Nannarelli is with the Department of Applied Mathematics and Computer Science, Technical University of Denmark, Kongens Lyngby 2800, Denmark (e-mail: alna@dtu.dk).

Color versions of one or more of the figures in this paper are available online at http://ieeexplore.ieee.org.

Digital Object Identifier 10.1109/TCSI.2016.2561518 set $\{-r / 2, \ldots,-1,0,1, \ldots, r / 2\}$ to reduce the complexity of digit multiplications. For $n$-bit operands, a total of $\lceil n / m\rceil$ partial products are generated for two's complement representation, and $\lceil(n+1) / m\rceil$ for unsigned representation.

Radix-4 modified Booth is a widely used recoding method, that recodes a binary operand into radix -4 signed digits in the set $\{-2,-1,0,1,2\}$. This is a popular recoding since the digit multiplication step to generate the partial products only requires simple shifts and complementation. The resulting number of partial products is about $n / 2$.

Higher radix signed recoding is less popular because the generation of the partial products requires odd multiples of the multiplicand which can not be achieved by means of simple shifts, but require carry-propagate additions. For instance, for radix-16 signed digit recoding [9] the digit set is $\{-8,-7, \ldots, 0, \ldots, 7,8\}$, so that some odd multiples of the multiplicand have to be generated. Specifically, it is required to generate $\times 3, \times 5$, and $\times 7$ multiples ( $\times 6$ is obtained by simple shift of $\times 3$ ). The generation of each of these odd multiplies requires a two term addition or subtraction, yielding a total of three carry-propagate additions.

However, the advantage of the high radix is that the number of partial products is further reduced. For instance, for radix-16 and $n$-bit operands, about $n / 4$ partial products are generated. Although less popular than radix-4, there exist industrial instances of radix-8 [10]-[16]. and radix-16 multipliers [17] in microprocessors implementations.

The choice of these radices is related to area/delay/power optimization of pipelined multipliers (or fused multiplier adder as in the case of a Intel Itanium microprocessor [17]), for balancing delay between stages and/or reduce the number of pipelining flip-flops.

A further consideration is that carry-propagate adders are today highly energy-delay optimized, while partial product reductions trees suffer the increasingly serious problems related to a complex wiring and glitching due to unbalanced signal paths. It is recognized in the literature that a radix-8 recoding leads to lower power multipliers compared to radix-4 recoding at the cost of higher latency (as a combinational block, without considering pipelining) [4], [18].

Moreover, although the radix-16 multiplier requires the generation of more odd multiples and has a more complex wiring for the generation of partial products [4], a recent microprocessor design [17] considered it to be the best choice for low power (under the specific constraints for this microprocessor).

In [1] and [2], some optimizations for radix-4 two's complement multipliers were introduced. Although for $n$-bit operands, a total of $\lceil n / 2\rceil$ partial products are generated, the resulting 
maximum height of the partial product array is $\lceil n / 2\rceil+1$ elements to be added (in just one of the columns). This extra height by a single-bit row is due to the +1 introduced in the bit array to make the two's complement of the most significant partial product (when the recoded most significant digit of the multiplier is negative). The maximum column height may determine the delay and complexity of the reduction tree [7], [16]. In [1] and [2], authors showed that this extra column of one bit could be assimilated (with just a simplified three bit addition) with the most significant part of the first partial product without increasing the critical path of the recoding and partial product generation stage. The result is that the partial product array has a maximum height of $\lceil n / 2\rceil$.

This reduction of one bit in the maximum height might be of interest for high-performance short-bit width two's complement multipliers (small $n$ ) with tight cycle time constraints, that are very common in SIMD digital signal processing applications. Moreover, if $n$ is a power of two, the optimization allows to use only 4-2 carry-save adders for the reduction tree, potentially leading to regular layouts [16].

These kind of optimizations can become particularly important as they may add flexibility to the "optimal" design of the pipelined multiplier. Optimal pipelining in fact, is a key issue in current and future multiplier (or multiplier-add) units: 1) the latency of the pipelined unit is very important, even for throughput oriented applications, as it impacts the energy consumption of the whole core [19]; and 2) the placement of the pipelining flip-flops should at the same time minimize total power, due to the number of flip-flops required and the unbalanced signal propagation paths.

The methods proposed in [1] and [2] were mostly focused on two's complement radix-4 Booth multipliers, thus leaving open the research and extension to higher radices and unsigned multiplications (for unsigned integer arithmetic or mantissa times mantissa in a floating-point unit). For a radix higher than 4 , it is necessary to generate the odd multiples (usually with adders), resulting in the reduction of the time slacks necessary to "hide" the simplified three bit assimilation. Unsigned multiplication may produce a positive carry out during recoding (this depends of the value of $n$ and the radix used for recoding), leading to one additional row, increasing the maximum height of the partial product array by one row, not just in one but in several columns. For all these reasons, we need to extend the techniques presented in [1] and [2].

In this work, we present a technique that allows partial product arrays of maximum height of $[n / m]$ (with the goal of not increasing the delay of the partial product generation stage), for $r>4$ and unsigned multipliers. Since for the standard unsigned multiplier the maximum height is $\lceil(n+1) / m\rceil$, the proposed method allows a reduction of one row when $n$ is a multiple of $m$. Our technique is general, but its impact (reduction of one row without increasing the critical path of the partial product generation stage) depends on the specific timing of the different components. Therefore, we can not claim a successful result for all practical values of $r$ and $n$ and different implementation technologies. Thus, we concentrate on an specific instance: a 64-bit radix-16 Booth recoded unsigned multiplier implemented with a synthesis tool and a standard-cell library. We use radix-16 since it is the most complex case, among the practical values of the radix, for the design of our scheme. The unsigned multiplier is also more complex for the design of our scheme than the signed multiplier. We use 64 bits, since it is a representative large wordlength. The method proposed can be adapted easily to other instances (signed, combined unsigned/signed, radix-8 recoding, different values of $n$ ).

The paper is structured as follows. Section II reviews the basic radix-16 Booth recoded multiplier. Section III describes the proposed method. Section IV presents the evaluation of our scheme, and Section V is devoted to the final conclusions.

\section{BASIC RADIX-16 Booth Multiplier}

In this section, we describe briefly the architecture of the basic radix-16 Booth multiplier (see [17] for instance). For sake of simplicity, but without loss of generality, we consider unsigned operands with $n=64$.

Let us denote with $\mathrm{X}$ the multiplicand operand with bit components $x_{i}$ ( $i=0$ to $n-1$, with the least-significant bit, LSB, at position 0) and with Y the multiplier operand and bit components $y_{i}$.

The first step is the recoding of the multiplier operand [8]: groups of four bits with relative values in the set $\{0,1, \ldots$, $14,15\}$ are recoded to digits in the set $\{-8,-7, \ldots, 0, \ldots$, $7,8\}$ (minimally redundant radix-16 digit set to reduce the number of multiples).

This recoding is done with the help of a transfer digit $t_{i}$ and an interim digit $w_{i}$ [7]. The recoded digit $z_{i}$ is the sum of the interim and transfer digits

$$
z_{i}=w_{i}+t_{i}
$$

When the value of the four bits, $v_{i}$, is less than 8 , the transfer digit is zero and the interim digit $w_{i}=v_{i}$. For values of $v_{i}$ greater than or equal to $8, v_{i}$ is transformed into $v_{i}=16-\left(16-v_{i}\right)$, so that a transfer digit is generated to the next radix-16 digit position $\left(t_{i+1}\right)$ and an interim digit of value $w_{i}=-(16-v)$ is left. That is

$$
\begin{aligned}
& 0 \leq v_{i}<8: t_{i+1}=0 \quad w_{i}=v_{i} \quad w_{i} \in[0,7] \\
& 8 \leq v_{i} \leq 15: t_{i+1}=1 \quad w_{i}=-\left(16-v_{i}\right) \quad w_{i} \in[-8,-1] .
\end{aligned}
$$

The transfer digit corresponds to the most-significant bit (MSB) of the four-bit group, since this bit determines if the radix-16 digit is greater than or equal to 8 . The final logical step is to add the interim digits and the transfer digits ( 0 or 1$)$ from the radix-16 digit position to the right.

Since the transfer digit is either 1 or 0 , the addition of the interim digit and the transfer digit results in a final digit in the set $\{-8,-7, \ldots, 0, \ldots, 7,8\}$. Due to a possible transfer digit from the most significant radix-16 digit, the number of resultant radix- 16 recoded digits is $\lceil(n+1) / 4\rceil$. Therefore, for $n=64$ the number of recoded digits (and the number of partial products) is 17. Note that the most significant digit is 0 or 1 because it is in fact just a transfer digit.

After recoding, the partial products are generated by digit multiplication of the recoded digits times the multiplicand X. 


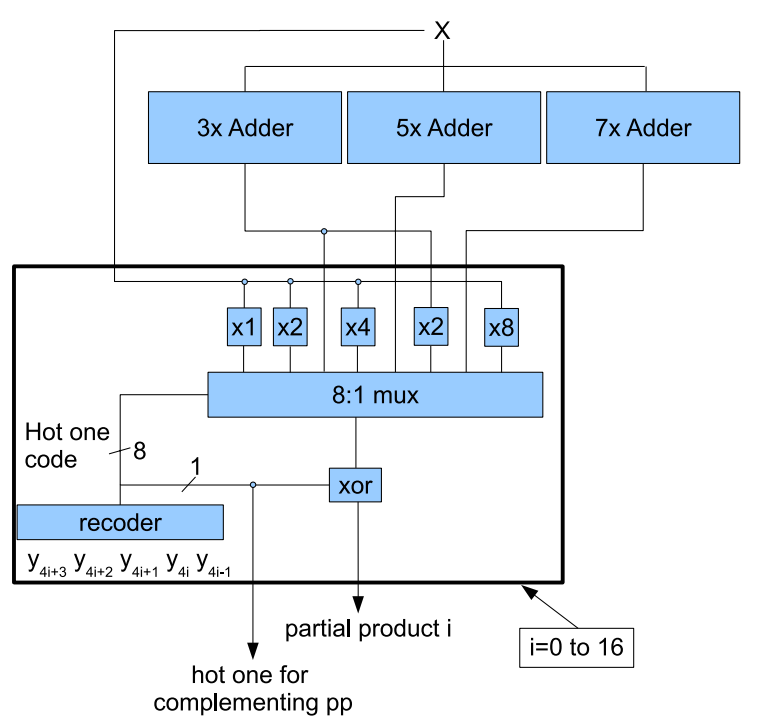

Fig. 1. Partial product generation.

For the set of digits $\{-8,-7, \ldots, 0, \ldots, 7,8\}$, the multiples $1 \mathrm{X}, 2 \mathrm{X}, 4 \mathrm{X}$, and $8 \mathrm{X}$ are easy to compute, since they are obtained by simple logic shifts. The negative versions of these multiples are obtained by bit inversion and addition of a 1 in the corresponding position in the bit array of the partial products. The generation of $3 \mathrm{X}, 5 \mathrm{X}$, and $7 \mathrm{X}$ (odd multiples) requires carry-propagate adders (the negative versions of these multiples are obtained as before). Finally, $6 \mathrm{X}$ is obtained by a simple one bit left shift of $3 \mathrm{X}$.

Fig. 1 illustrates a possible implementation of the partial product generation. Five bits of the multiplier $\mathrm{Y}$ are used to obtain the recoded digit (four bits of one digit and one bit of the previous digit to determine the transfer digit to be added). The resultant digit is obtained as a one-hot code to directly drive a 8 to 1 multiplexer with an implicit zero output (output equal to zero when all the control signals of the multiplexer are zero). The recoding requires the implementation of simple logic equations that are not in the critical path due to the generation in parallel of the odd multiples (carry-propagate addition). The $\mathrm{XOR}$ at the output of the multiplexer is for bit complementation (part of the computation of the two's complement when the multiplier digit is negative).

Fig. 2(a) illustrates part of the resultant bit array for $n=64$ after the simplification of the sign extension [7].

In general, each partial product has $n+4$ bits including the sign in two's complement representation. The extra four bits are required to host a digit multiplication by up to 8 and a sign bit due to the possible multiplication by negative multiplier digits.

Since the partial products are left-shifted four bit positions with respect to each other, a costly sign extension would be necessary. However, the sign extension is simplified by concatenation of some bits to each partial product ( $S$ is the sign bit of the partial product and $C$ is $S$ complemented): $C S S S$ for the first partial product and $111 C$ for the rest of partial products (except the partial product at the bottom that is non negative since the corresponding multiplier digit is 0 or 1 ). The bits denoted by $b$ in Fig. 2 corresponds to the logic 1 that is added for the two's complement for negative partial products.
After the generation of the partial product bit array, the reduction (multioperand addition) from a maximum height of 17 (for $n=64$ ) to 2 is performed. The methods for multioperand addition are well known, with a common solution consisting of using 3 to 2 bit reduction with full adders (or 3:2 carry-save adders) or 4 to 2 bit reduction with 4:2 carry-save adders.

The delay and design effort of this stage are highly dependent on the maximum height of the bit array. It is recognized that reduction arrays of 4:2 carry-save adders may lead to more regular layouts [16]. For instance, with a maximum height of 16, a total of 3 levels of 4:2 carry-save adders would be necessary. A maximum height of 17 leads to different approaches that may increase the delay and/or require to use arrays of 3:2 carry-save adders interconnected to minimize delay [20].

After the reduction to two operands, a carry-propagate addition is performed. This addition may take advantage of the specific signal arrival times from the partial product reduction step.

\section{Proposed Method}

To reduce the maximum height of the partial product bit array we perform a short carry-propagate addition in parallel to the regular partial product generation. This short addition reduces the maximum height by one row and it is faster than the regular partial product generation. Fig. 2(b) shows the elements of the bit array to be added by the short adder. Fig. 2(c) shows the resulting partial product bit array after the short addition. Comparing both figures, we observe that the maximum height is reduced from 17 to 16 for $n=64$.

Fig. 3 shows the specific elements of the bit array (boxes) to be added by the short carry-propagate addition. In this figure, $p_{i, j}$ corresponds to the bit $j$ of partial product $i, s_{0}$ is the sign bit of partial product $0, c_{0}=\operatorname{NOT}\left(s_{0}\right), b_{i}$ is the bit for the two's complement of partial product $i$, and $z_{i}$ is the $i$ th bit of the result of the short addition.

The selection of these specific bits to be added is justified by the fact that, in this way, the short addition delay is hidden from the critical path that corresponds to a regular partial product generation (this will be shown in Section IV). We perform the computation in two concurrent parts $\mathrm{A}$ and $\mathrm{B}$ as indicated in Fig. 3. The elements of the part A are generated faster than the elements of part B. Specifically the elements of part A are obtained from:

- the sign of the first partial product: this is directly obtained from bit $y_{3}$ since there is no transfer digit from a previous radix-16 digit;

- bits 3 to 7 of partial product 16: the recoded digit for partial product 16 can only be 0 or 1 , since it is just a transfer digit. Therefore the bits of this partial product are generated by a simple AND operation of the bits of the multiplicand $\mathrm{X}$ and bit $y_{63}$ (that generates the transfer from the previous digit).

Therefore, we decided to implement part A as a speculative addition, by computing two results, a result with carry-in $=0$ and a result with carry-in $=1$. This can be computed efficiently with a compound adder [7]. Fig. 4 shows the implementation of part A. The compound adder determines speculatively the 


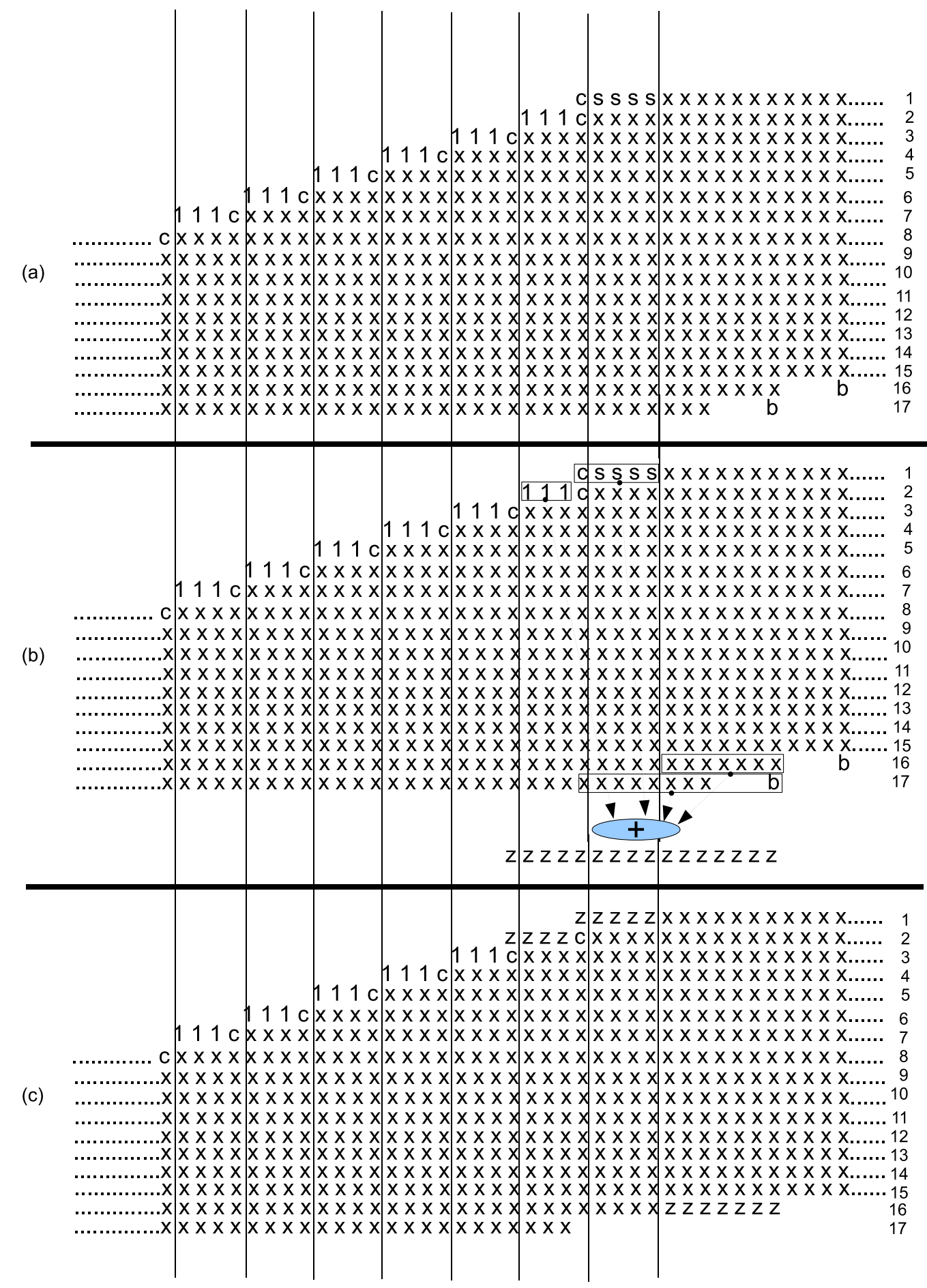

Fig. 2. Radix-16 partial product reduction array.

two possible results. Once the carry-in is obtained (from part B), the correct result is selected by a multiplexer. Note that the compound adder is of only five bits, since the propagation of the carry through the most significant three ones is straightforward.

The computation of part B is more complicated. The main issue is that we need the 7 least-significant bits of partial product 15 . Of course waiting for the generation of partial product 15 is not an option since we want to hide the short addition delay out of the critical path. We decided to implement a specific circuit to embed the computation of the least-significant

\begin{tabular}{|c|c|c|}
\hline \multicolumn{3}{|c|}{ A } \\
\hline $\begin{array}{r}111 \\
+\end{array}$ & $\begin{array}{lllll}c_{0} & s_{0} & s_{0} & s_{0} & s_{0} \\
p_{16,7} & p_{16,6} & p_{16,5} & p_{16,4} & p_{16,}\end{array}$ & $\begin{array}{|lll|}p_{15,6} p_{15,5} p_{15,4} & p_{15,3} p_{15,2} & p_{15,1} \\
p_{15,2} & p_{15,0} \\
p_{16,2} & p_{16,1} p_{16,0} & b_{15}\end{array}$ \\
\hline$z_{15} z_{14} z_{13} z_{12}$ & $\begin{array}{lllll}Z_{11} & Z_{10} & Z_{9} & Z_{8} & Z_{7}\end{array}$ & \begin{tabular}{|lllllll}
$z_{6}$ & $z_{5}$ & $z_{4}$ & $z_{3}$ & $z_{2}$ & $z_{1}$ & $z_{0}$
\end{tabular} \\
\hline
\end{tabular}

Fig. 3. Detail of the elements to be added by the short addition.

bits of partial product 15 in the computation of part B (and also the addition of the bit $b_{15}$ ). Note that for the method to be correct the computation of the partial product embedded in 


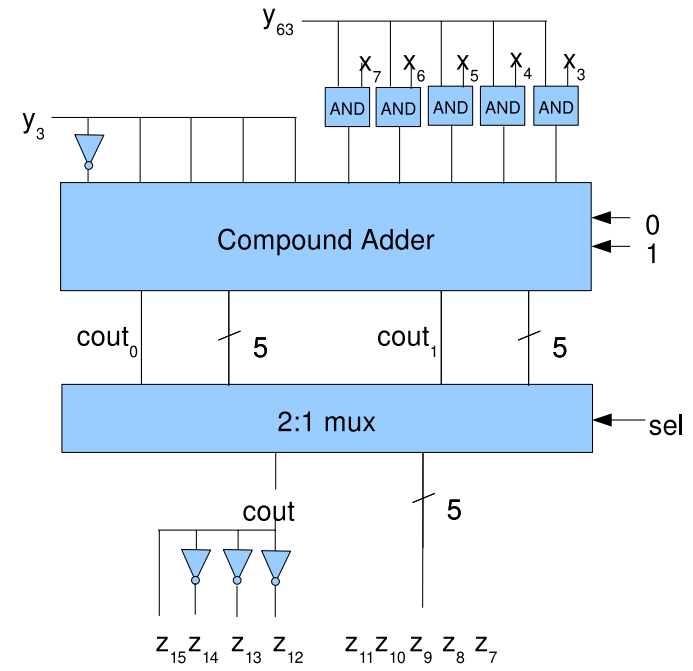

Fig. 4. Speculative addition of part A.

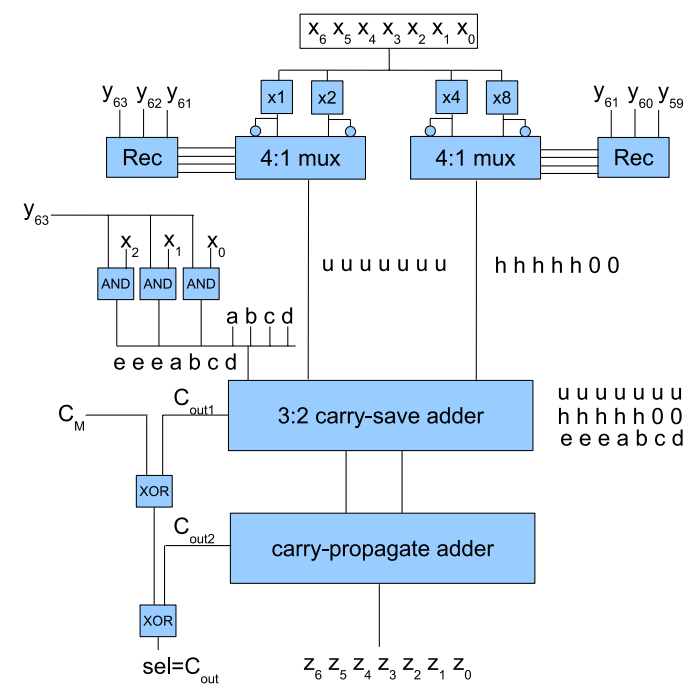

Fig. 5. Computation of part B.

part B should be consistent with the regular computation performed for the most significant bits of partial product 15 .

Fig. 5 shows the computation of part B. We decided to compute part B as a three operand addition with a $3: 2$ carrysave adder and a carry-propagate adder. Two of the operands correspond to the least-significant bits of the partial product 15 and the other operand corresponds to the three least-significant bits of partial product 16 (that are easily obtained by an AND operation). We perform the computation of the bits of the radix-16 partial product 15 as the addition of two radix-4 partial products. Therefore, we perform two concurrent radix-4 recodings and multiple selection. The multiples of the leastsignificant radix-4 digit are $\{-2,-1,0,1,2\}$, while the multiples for the most significant radix-4 digit are $\{-8,-4,0,4,8\}$ (radix-4 digit set $\{-2,-1,0,1,2\}$, but with relative weight of 4 with respect to the least-significant recoding). These two radix-4 recodings produce exactly the same digit as a direct radix-16 recoding for most of the bit combinations. However, among the 32 -bit combinations for a full radix-16 digit recoding, there are six not consistent with the two concurrent radix-4 recodings. Specifically:

- The bit strings 00100 and 11011 are recoded in radix-16 to 2 and -2 respectively. However, when performing two parallel radix -4 recodings the resulting digits are $(4,-2)$ and $(-4,2)$ respectively. That is, the radix-4 recoding performs the computation of $2 \mathrm{X}(-2 \mathrm{X})$ as $4 \mathrm{X}-2 \mathrm{X}$ $(-4 \mathrm{X}+2 \mathrm{X})$. To have a consistent computation we modified the radix- 4 recoders so that these strings produce radix-4 digits of the form $(0,2)$ and $(0,-2)$.

- The bit strings 00101 and 00110 are recoded in radix-16 to 3 in both cases. However, the resulting radix- 4 digits are $(4,-1)$. This means that the radix-4 recoding performs the computation of $3 \mathrm{X}$ as $4 \mathrm{X}-\mathrm{X}$.

To address this inconsistency problem, in this case, we decided to implement the radix-16 multiple $3 \mathrm{X}$ as $4 \mathrm{X}-\mathrm{X}$. This avoids the combination of radix-4 digits $(2,1)$ and simplifies the multiplexers in Fig. 5.

- The bit strings 11001 and 11010 are recoded in radix-16 to -3 in both cases. However, the resulting radix-4 digits are $(-4,1)$. Therefore, for consistency, we proceed as in the previous case by generating the radix- 16 multiple $-3 \mathrm{X}$ as $-4 \mathrm{X}+\mathrm{X}$.

To handle negative multiples, we select complemented inputs in the multiplexers and place 1 in a slot of the input of the $3: 2$ carry-save adder with relative binary weight equal to the absolute value of the corresponding radix- 4 digit. These hot ones for two's complement are indicated in Fig. 5 as the string "abcd." For instance, if the least-significant radix-4 digit is -2 and the most significant radix-4 digit is -4 , then $c=1$ and $b=1$. Therefore, "abcd" signals are obtained directly from the selection bits of the $4: 1$ multiplexers.

Fig. 6 shows the recoding and partial product generation stage including the high level view of the hardware scheme proposed.

The way we compute part B may still lead to an inconsistency with the computation of the most significant part of partial product 15 . Specifically, when partial product 15 is the result of an odd multiple, a possible carry from the 7 least-significant bits is already incorporated in the most significant part of the partial product. During the computation of part B we should not produce again this carry. This issue is solved as follows.

Let us consider first the case of positive odd multiples. Fig. 5 shows that the computation of part B may generate two carry outs: the first from the 3:2 carry-save adder $\left(C_{\text {out1 } 1}\right)$, and the second from the carry-propagate adder $\left(C_{\text {out } 2}\right)$. To avoid inconsistencies, we detect the carry propagated to the most significant part of the partial product 15 (we call this $C_{M}$ ) and subtract it from the two carries generated in part B.

Specifically, Table I shows the truth table to generate the carry out of part B. This truth table corresponds to the XOR of the three inputs. The $C_{M}$ carry is obtained from a multiplexer that selects among the carry to bit position 7 from the odd multiple generators $(\times 3, \times 5$, and $\times 7)$, the carry to bit position 6 from the multiple generator $\times 3$ (to get the carry to position 7 of multiple $\times 6$ ), or carry zero for the other multiples. The resultant carry out is the selection signal used in the multiplexer of part A. 


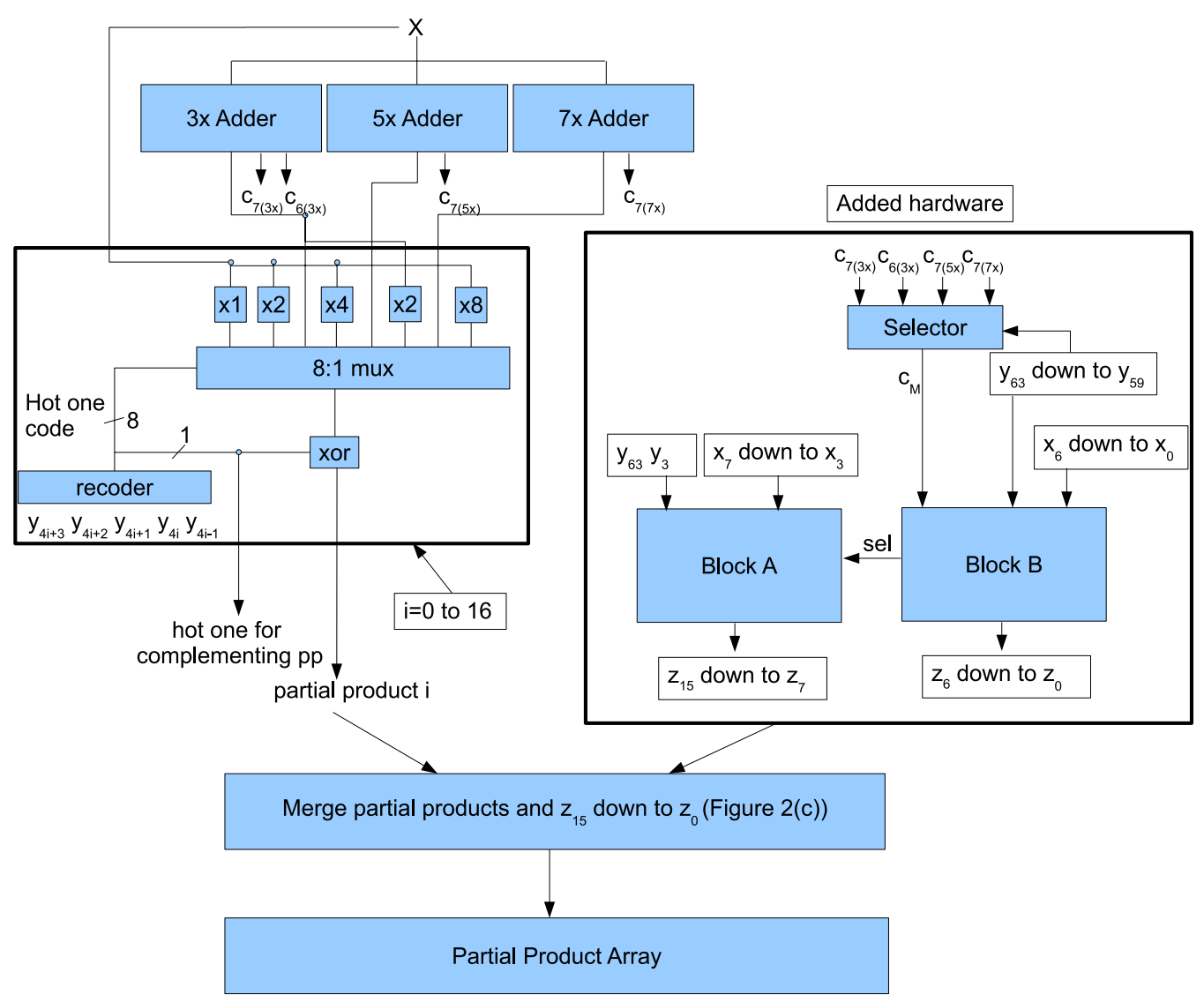

Fig. 6. High level view of the recoding and partial product generation stage including our proposed scheme.

TABLE I

TRUTH TABLE FOR COMPUTING THE CARRY OUT (- STANDS FOR "DON'T CARE")

\begin{tabular}{|ccc||c|}
\hline$C_{M}$ & $C_{\text {out } 1}$ & $C_{\text {out } 2}$ & $C_{\text {out }}$ \\
\hline \hline 0 & 0 & 0 & 0 \\
\hline 0 & 0 & 1 & 1 \\
\hline 0 & 1 & 0 & 1 \\
\hline 0 & 1 & 1 & - \\
\hline 1 & 0 & 0 & - \\
\hline 1 & 0 & 1 & 0 \\
\hline 1 & 1 & 0 & 0 \\
\hline 1 & 1 & 1 & 1 \\
\hline
\end{tabular}

For negative odd multiples we use a similar scheme. In this case the output of adder is complemented, but the only information available about the carry to position 7 is obtained directly from the adders that generate the positive odd multiple.

Next, we show how to obtain the carry to the most significant part of the resultant complemented odd multiple from the carry to position 7 obtained from the adders.

Let us call $M$ the result of the positive odd multiple (output of the adder), and express $M$ as

$$
M=N+P
$$

with $P$ being the seven least-significant bits of the result from the adder, and $N$ the remaining most significant bits of the result of the adder. Let us express $N$ in terms of $C_{7}$ (carry to position 7)

$$
N=Q+C_{7} 2^{7}
$$

that is, $Q$ are the remaining most significant bits of the positive odd multiple minus the carry to position 7 . Assuming a $m$ bit partial product, the complement of $M$ is expressed as

$$
\bar{M}=2^{n}-1-M=2^{n}-1-N-C_{7} 2^{7}-Q .
$$

By adding and subtracting $2^{7}$ and rearranging terms results in

$$
\bar{M}=2^{n}-2^{7}-N-C_{7} 2^{7}+2^{7}-1-Q .
$$

We identify the terms $\bar{N}=2^{n}-2^{7}-N$ and $\bar{Q}=2^{7}-1-Q$. Taking into account these terms and adding and subtracting $2^{7}$ and $2^{n-1}$ results in

$$
\bar{M}=-2^{n-1}+\bar{N}+\left(2^{n-1}-2^{7}\right)+\left(1-C_{7}\right) 2^{7}+\bar{Q} .
$$

The term $\left(1-C_{7}\right) 2^{7}+\bar{Q}=\overline{C_{7}}+\bar{Q}$ is computed in part B of the proposed scheme (see Fig. 5), but $\left(1-C_{7}\right) 2^{7}=\overline{C_{7}}$ is also part of the most significant part of partial product 15 . Therefore, for a negative partial product we need to subtract $\overline{C_{7}}$.

In summary, we take $C_{M}$ as the carry to position 7 of the adder that generates the multiple when the partial product is positive, and complement this carry, when the partial product is negative.

\section{EvAluATION}

In this section we evaluate the proposed method. The main goal of this section is to demonstrate that with current technologies, it is possible to "hide" the delay of the additional logic 


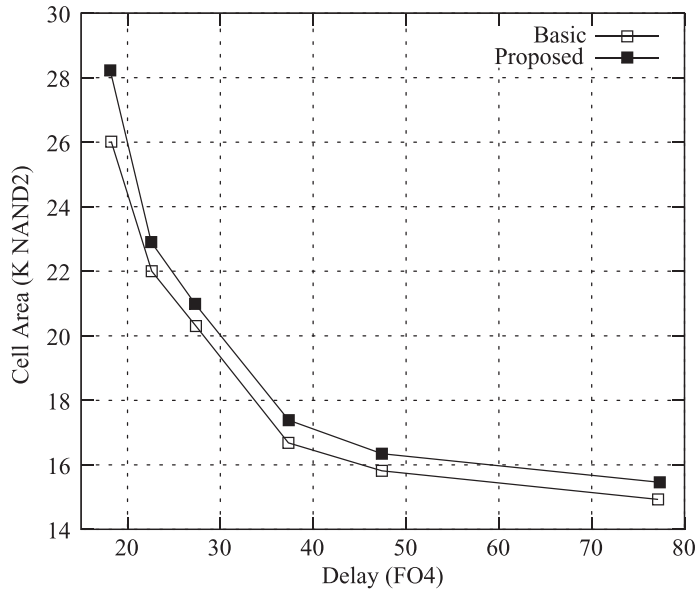

Fig. 7. Latency-area space for the partial product generation stage: basic scheme vs proposed scheme.

placed in parallel to the partial product generation, so that it is out of the critical path.

First, we show the results of the hardware synthesis using state of the art CAD tools (Synopsys Design Compiler [21]). Second, we evaluate the impact of the proposed method on the whole multiplier for different pipeline choices. Then, we show a technology independent path analysis using a high level areadelay model to have more insight in the component delays of the critical path.

\section{A. Synthesis With CAD Tools}

We have performed a hardware synthesis using Synopsys Design Compiler [21] with the STM 90nm CMOS standard cell library. For this library the delay of a FO4 is 45 ps (FO4 is the delay of an inverter of minimum size with a load of four inverters), and the area of a two-input NAND gate is $4.4 \mu \mathrm{m}^{2}$. We synthesized the full partial product generation stage for the basic scheme allowing Synopsys' DesignWare [21] to choose the adder, and the proposed scheme with hand coding of adders (we need the internal carry of the adders, so we were not able to use DesignWare in this case). We did not optimize the $3 \mathrm{X}$ adder as described for instance in [12], [22] and [23], since this optimization can not be applied to the $5 \mathrm{X}$ and $7 \mathrm{X}$ adders, so that the critical path remains the same.

Fig. 7 shows the latency-area space for the two synthesized designs. For higher latency points, as expected, the proposed design has a slight increase in area. The fastest design point is roughly the same for the two designs, although the proposed design has a penalty of about $2 \mathrm{~K}$ additional NAND-2 gates with respect to the basic scheme. For the fastest design point, the cost of the additional hardware in the proposed scheme is about 500 NAND-2 gates (even less since 7 least-significant bits of one radix-16 regular partial product are not required), less than $1.8 \%$ of the hardware complexity of the partial product generation stage. Therefore, the extra $1.5 \mathrm{~K}$ NAND-2 gates corresponds to the penalty of not using DesignWare adders in the proposed design.

Our synthesis experiment shows that the proposed scheme does not introduce any significant variation in the latency-area

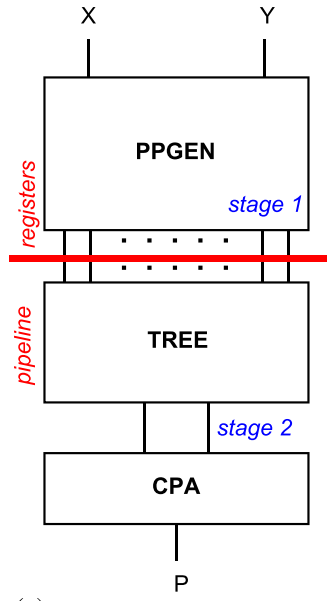

(a)

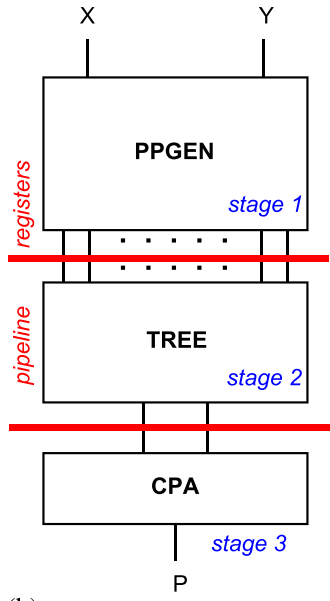

(b)
Fig. 8. Pipelined multiplier: (a) 2-stage; (b) 3-stage.

space of the partial product generation stage, confirming our hypothesis that the introduced hardware has a minor cost and is hidden from the critical path. Therefore, we have the benefit of reducing the maximum height of the partial product array by one unit without introducing any significant penalties in the partial product generation stage.

\section{B. Impact on the Multiplier}

In the previous subsection, we provided the detail of the synthesis of the partial product generation with the proposed method. In this subsection, we evaluate the impact of our method on the whole multiplier. We implement a multiplier by the proposed method to reduce the partial products by one, and we compare its performance (maximum clock frequency, area and power dissipation) to a multiplier, referred as basic, with the standard partial product generation and an extra operand in the accumulation tree.

A practical design of a $64 \times 64$ multiplier is normally pipelined to guarantee high-throughput. However, the placement of pipeline registers depends mostly on the specific technology and may vary from design to design. High radix multipliers are chosen because the shallower trees allow a significant power reduction, since the glitching power is limited to a few levels of gates in the tree.

For this reason, it is realistic to place pipeline registers before the tree, i.e., store the partial products in the pipeline registers.

Consequently, we evaluate two schemes:

1) a 2-stage pipelined design [see Fig. 8(a)]with pipeline register placed between the partial products generation (stage abbreviated as PPGEN in the figures and tables) and the tree (TREE);

2) a 3-stage design [see Fig. 8(b)] with an additional pipeline register placed between the tree and the final carrypropagate adder (CPA).

Other pipeline placements are not convenient because they will result in placing flip-flops inside functional units, such as CPAs or adder trees. This may result in increased number of flip-flops (e.g., inside the tree) and it is also nonsuitable for reuse. Standard datapath blocks (e.g., CPAs) are normally taken 


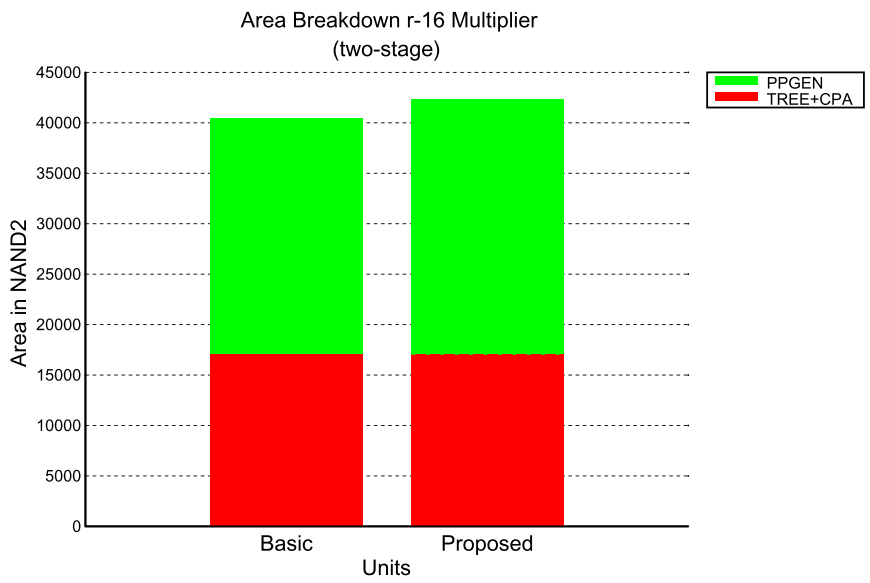

Fig. 9. Area breakdown for 2-stage pipelined multipliers.

TABLE II

POWER DisSIPATION IN THE PIPELINED MULTIPLIERS

\begin{tabular}{|l|rr|rr||rr|rr|}
\hline & \multicolumn{3}{|c||}{ TWO-STAGE } & \multicolumn{4}{c|}{ THREE-STAGE } \\
& \multicolumn{2}{|c|}{ BASIC } & \multicolumn{2}{|c|}{ PROPOSED } & \multicolumn{2}{c|}{ BASIC } & \multicolumn{2}{|c|}{ PROPOSED } \\
PPG] & [mW & [mW] & $\%$ & {$[\mathrm{~mW}]$} & $\%$ & {$[\mathrm{~mW}$} & $\%$ \\
TREE & 1.93 & 28 & 1.95 & 29 & 3.29 & 46 & 2.97 & 43 \\
CPA & 2.44 & 35 & 2.29 & 34 & 1.29 & 18 & 1.27 & 19 \\
REGs & 0.51 & 7 & 0.47 & 7 & 0.23 & 3 & 0.23 & 3 \\
\hline TOTAL & 1.99 & 29 & 2.02 & 30 & 2.34 & 33 & 2.37 & 35 \\
\hline
\end{tabular}

Power is measured at $100 \mathrm{MHz}$ frequency.

from fully-tested hardware libraries and altering their behavior (placing pipeline registers inside) will prolong development times, revalidation and retesting.

1) Design of 2-Stage Multiplier: For the 2-stage multiplier the critical path lies in the second stage for both the basic and the proposed multipliers. The delay of the critical path is 23 FO4 for the basic and 21.5 FO4 for the proposed multiplier.

Clearly, the reduced number of partial products in the proposed unit at the tree input (16 versus 17 operands) makes the accumulation faster. The area of the 2-stage implementation it is slightly larger for the proposed multiplier, as shown in Fig. 9.

As for the power dissipation, Table II reports the power breakdown for the main blocks of the pipelined multiplier. The proposed unit consumes about $2 \%$ less power than the basic unit. This is mostly due to the reduced switching activity (glitches) in the second stage (tree and CPA).

2) Design of 3-Stage Multiplier: The maximum throughput for the multiplier can be obtained by breaking the critical part of the second stage in two stages. To minimize the number of flip-flops, or latches, this second register is placed between the tree and the CPA.

With this pipelining, the critical path lies in the first stage of the multiplier for both the basic and the proposed multipliers.

As already shown in Section IV-A, the delay of the critical path is $18 \mathrm{FO} 4$ for both implementations.

In this case, the larger slack ${ }^{1}$ in stage 2 , allows for a good reduction in area for the tree of the proposed multiplier, that partly

\footnotetext{
${ }^{1}$ The synthesizer trades-off slack for smaller area and lower power dissipation gates. Therefore, the actual slack reported by the static timing analysis is close to zero for the three stages.
}

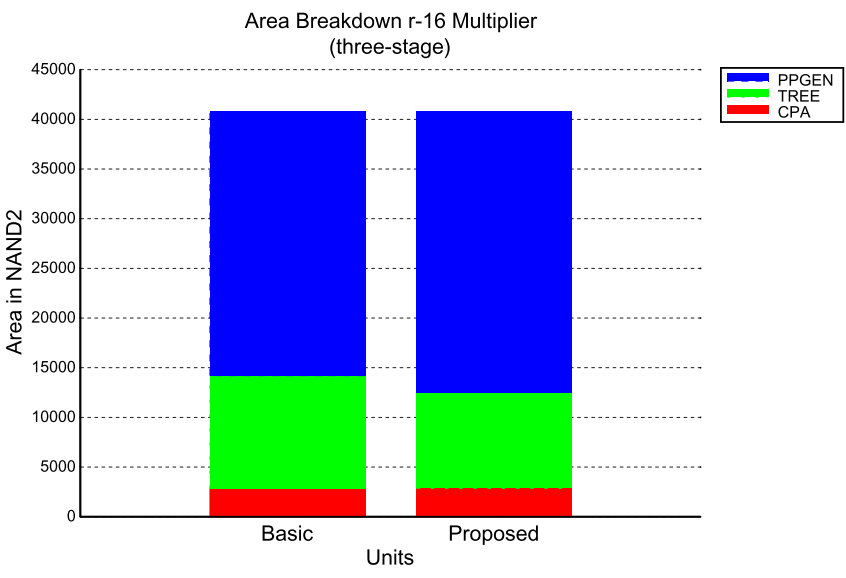

Fig. 10. Area breakdown for 3-stage pipelined multipliers.

TABLE III

DElay EQUations, INPUT CAPACITANCE AND HARDWARE COST OF BASIC ElEMENTS

\begin{tabular}{|c|c|c|c|}
\hline Element & $\begin{array}{c}\text { Delay } \\
\text { \# FO4 }\end{array}$ & $\begin{array}{c}\text { Input Capacitance } \\
\text { \# inverters }\end{array}$ & $\begin{array}{c}\text { Area } \\
\text { \# NAND2 }\end{array}$ \\
\hline NAND2 & $0.4+0.2 L$ & $4 / 3$ & 1.0 \\
\hline NOR2 & $0.4+0.2 L$ & $5 / 3$ & 1.3 \\
\hline INV & $0.2+0.2 L$ & 1 & 0.4 \\
\hline AOI12 & $0.47+0.2 L$ & $(5 / 3,2,2)$ & 2.1 \\
\hline OAI12 & $0.53+0.2 L$ & $(4 / 3,2,2)$ & 2.0 \\
\hline XOR & $0.9+0.2 L$ & $7 / 3$ & 2.5 \\
\hline MUX2 & $0.9+0.2 L$ & $($ data $: 4 / 3$, sel $: 7 / 3)$ & 2.5 \\
\hline MUX4 & $1.5+0.2 L$ & $($ data $: 4 / 3$, sel $: 7 / 3)$ & 5.0 \\
\hline Full-Adder & $a, b: 2.73+0.2 L$ & $(a: 11 / 3, b: 7 / 3)$ & 7.5 \\
& $c: 0.9+0.2 L$ & $11 / 3$ & \\
\hline Buffer & $0.72 \ln \left(L / L_{\text {in }}\right)$ & $L_{\text {in }}$ & $\ln \left(L / L_{\text {in }}\right)$ \\
\hline
\end{tabular}

compensate the larger area in the first stage (see Fig. 7). As a result, the area of the two units in the 3-stage implementation is almost the same, as reported in Fig. 10.

Also in this case, the power dissipation is slightly $(4 \%)$ lower in the proposed unit. The breakdown of the different parts is reported in Table II.

\section{High Level Evaluation}

In this subsection we use a high level rough model to evaluate the proposed method. We evaluate the critical path of the conventional partial product generation and the critical path of the hardware we added to reduce the maximum height of the partial product array. Although real implementations rely on optimizations of the critical path done by synthesis tools on a specific standard cell library technology, this high level analysis may give some insight about the relative contribution to the critical path of each component.

We use a rough delay model based on logical effort [24]. This model is based on using cells with transistor sizing so that all the cells have the drive strength of the minimum size inverter. Buffering is introduced when necessary to optimize delays. We provide delays in FO4 units. Interconnections loads are not taken into account. Optimizations such as gate sizing, low/high $\mathrm{V}_{\mathrm{th}}$, etc. are not considered.

Table III shows the delay equations, input capacitance and relative hardware cost of the basic hardware elements used. In the 


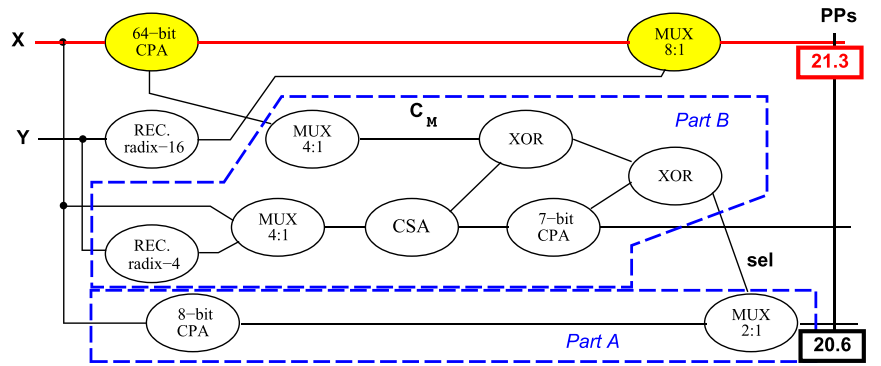

Fig. 11. Timing paths for the proposed partial products reduction.

table, the parameter $L$ indicates the actual load (capacitance) connected to the specific gate, and $L_{\text {in }}$ indicates the input capacitance of the buffers.

A key issue for the estimation of the critical path of the conventional partial product generation is the architecture of the adders for multiple generation. The worst case for our analysis corresponds to the fastest design point for partial product generation. Therefore we considered a fast Kogge-Stone adder topology [7]. Although this is not energy/power efficient in real implementations, at the logic level it is a good lower bound of delay for an adder.

After the analysis of the conventional architecture, we estimated the impact of the additional hardware required for the proposed multiplier. For a quick reference, the timing paths of Figs. 4-6 are summarized in Fig. 11. In the figure, the delay in the input registers ( $\mathrm{X}$ and $\mathrm{Y}$ ) and the delay of buffers are omitted for simplicity.

The critical path of the conventional partial product generation is composed by the following items:

- input register X: 3.0 FO4;

- input buffering of multiplicand: $1.4 \mathrm{FO} 4$;

- multiple generation (adder): 10.3 FO4;

- buffer between multiple generators and 8:1 mux: 1.7 FO4;

- 8:1 mux and inversion (input from data): 4.9 FO4.

This corresponds to a total delay of $21.3 \mathrm{FO} 4$ in the critical path.

The scheme we propose (Part A, Fig. 4, and Part B, Fig. 5, in Fig. 11) has the following components in the critical path:

- input register Y: $3.0 \mathrm{FO}$;

- input buffering of multiplier bits: $0.5 \mathrm{FO} 4$;

- radix-4 Booth recoding and selector with inversion (Part B): 5.0 FO4;

- 3:2 carry-save adder (Part B): 3.5 FO4;

- carry out of 7-bit carry-propagate adder (Part B): 4.4 FO4;

- XOR to produce sel signal (Part B) and six-bit 2:1 multiplexer (Part A): 4.2 FO4.

Thus, the path delay is 20.6 FO4 and it is not critical.

Our analysis shows that the $C_{M}$ signal is not in the critical path (the worst case delay for $C_{M}$ is $13.1 \mathrm{FO}$, while the worst case delay for $C_{\text {out2 } 2}$ is $16.4 \mathrm{FO} 4$ ).

These results are coherent with the fastest design point in the latency-area graph shown in Fig. 7. The lower bound in latency is about $18.2 \mathrm{FO}$. The synthesis tool is able to do some sort of gate sizing (dependent on the available gate sizes for each instance gate), so a faster result than in our high level analysis should be expected.

Therefore, our rough analysis is in agreement with the synthesis results, as the proposed scheme is not in the critical path for $n=64$.

We performed a similar analysis for the critical path of the conventional partial product generation for $n=32$ (the case for $n=16$ is less attractive for radix-16 due to the small number of partial products). For $n=32$ we obtain a critical path of 19.7 FO4. As it can be seen in Fig. 2, the scheme we propose is not sensitive to the variation of $n$ (the number of bits added in Fig. 2(b) is independent of the value of $n$ ), thus resulting in the same critical path as before (20.6 FO4). Therefore, for the fastest design point, for $n=32$, the proposed scheme is in the critical path, with a slack with respect to the conventional partial product generation of $0.9 \mathrm{FO} 4$ for $n=32$. This negative slack of our scheme can be reduced with conventional approaches like low Vth gates and gate sizing without significant increase in power, since the share of our scheme with respect to the total hardware is very small.

We verified this statement with the synthesis tool. A synthesis for $n=32$ leads to a critical path of $16.5 \mathrm{FO} 4$ and this critical path corresponds to the computation of a regular partial product.

\section{CONCLUSION}

Pipelined large wordlength digital multipliers are difficult to design under the constraints of core cycle time (for nominal voltage), pipeline depth, power and energy consumption and area. Low level optimizations might be required to meet these constraints.

In this work, we have presented a method to reduce by one the maximum height of the partial product array for 64-bit radix-16 Booth recoded magnitude multipliers. This reduction may allow more flexibility in the design of the reduction tree of the pipelined multiplier. We have shown that this reduction is achieved with no extra delay for $n \geq 32$ for a cell-based design.

The method can be extended to Booth recoded radix- 8 multipliers, signed multipliers and combined signed/unsigned multipliers.

Radix-8 and radix-16 Booth recoded multipliers are attractive for low power designs, mainly to the lower complexity and depth of the reduction tree, and therefore they might be very popular in this era of power-constrained designs with increasing overheads due to wiring.

\section{REFERENCES}

[1] S. Kuang, J. Wang, and C. Guo, "Modified booth multipliers with a regular partial product array," IEEE Trans. Circuits Syst. II, Exp. Briefs, vol. 56, no. 5, pp. 404-408, May 2009.

[2] F. Lamberti et al., "Reducing the computation time in (short bit-width) twos complement multipliers," IEEE Trans. Comput., vol. 60, no. 2, pp. 148-156, Feb. 2011.

[3] N. Petra et al., "Design of fixed-width multipliers with linear compensation function," IEEE Trans. Circuits Syst. I, Reg. Papers, vol. 58, no. 5, pp. 947-960, May 2011.

[4] S. Galal et al., "FPU generator for design space exploration," in Proc. 21st IEEE Symp. Comput. Arithmetic (ARITH), Apr. 2013, pp. 25-34.

[5] K. Tsoumanis et al., "An optimized modified booth recoder for efficient design of the add-multiply operator," IEEE Trans. Circuits Syst. I, Reg. Papers, vol. 61, no. 4, pp. 1133-1143, Apr. 2014. 
[6] A. Cilardo et al., "High speed speculative multipliers based on speculative carry-save tree," IEEE Trans. Circuits Syst. I, Reg. Papers, vol. 61, no. 12, pp. 3426-3435, Dec. 2014.

[7] M. Ercegovac and T. Lang, Digital Arithmetic. Burlington, MA, USA: Morgan Kaufmann, 2004.

[8] S. Vassiliadis, E. Schwarz, and D. Hanrahan, "A general proof for overlapped multiple-bit scanning multiplications," IEEE Trans. Comput., vol. 38, no. 2, pp. 172-183, Feb. 1989.

[9] "Binary Multibit Multiplier," Patent 4745570 A, 1986.

[10] D. Dobberpuhl et al., "A 200-MHz 64-b dual-issue CMOS microprocessor," IEEE J. Solid-State Circuits, vol. 27, no. 11, pp. 1555-1567, Nov. 1992.

[11] E. M. Schwarz, R. M. A. III, and L. J. Sigal, "A radix-8 CMOS S/390 multiplier," in Proc. 13th IEEE Symp. Comput. Arithmetic (ARITH), Jul. 1997, pp. 2-9.

[12] J. Clouser et al., "A 600-MHz superscalar floating-point processor," IEEE J. Solid-State Circuits, vol. 34, no. 7, pp. 1026-1029, Jul. 1999.

[13] S. Oberman, "Floating point division and square root algorithms and implementation in the AMD-K7 microprocessor," in Proc. 14th IEEE Symp. Comput. Arithmetic (ARITH), Apr. 1999, pp. 106-115.

[14] R. Senthinathan et al., "A 650-MHz, IA-32 microprocessor with enhanced data streaming for graphics and video," IEEE J. Solid-State Circuits, vol. 34, no. 11, pp. 1454-1465, Nov. 1999.

[15] K. Muhammad et al., "Speed, power, area, latency tradeoffs in adaptive FIR filtering for PRML read channels," IEEE Trans. Very Large Scale Intgr. Syst., vol. 9, no. 1, pp. 42-51, Feb. 2001.

[16] G. Colon-Bonet and P. Winterrowd, "Multiplier evolution: A family of multiplier VLSI implementations," Comput. J., vol. 51, no. 5, pp. 585-594, 2008.

[17] R. Riedlinger et al., "A $32 \mathrm{~nm}, 3.1$ billion transistor, 12 wide issue itanium processor for mission-critical servers," IEEE J. Solid-State Circuits, vol. 47, no. 1, pp. 177-193, Jan. 2012.

[18] B. Cherkauer and E. Friedman, "A hybrid radix-4/radix-8 low power signed multiplier architecture," IEEE Trans. Circuits Syst. II, Analog Digit. Signal Process., vol. 44, no. 8, pp. 656-659, Aug. 1997.

[19] D. Lutz, "ARM FPUs: Low latency is low energy," presented at the 22nd IEEE Symposium in Computer Arithmetic, Jun. 2015, [last visited Jul. 1, 2016]. [Online]. Available: http://arith22.gforge.inria.fr/slides/s1-lutz.pdf

[20] V. G. Oklobdzija, D. Villeger, and S. S. Liu, "A method for speed optimized partial product reduction and generation of fast parallel multipliers using an algorithmic approach," IEEE Trans. Comput., vol. 45, no. 3, pp. 294-306, Mar. 1996.

[21] Synopsys Inc., "Design Compiler," [Online]. Available: http://www. synopsys.com

[22] "A X + 2 X Adder With Multi-Bit Generate/Propagate Circuit," Patent 5 875125,1997

[23] “3 × Adder," Patent 6269386 B1, 1998.

[24] A. Vazquez and E. Antelo, "Area and Delay Evaluation Model for CMOS Circuits,” Internal Report, Univ. Santiago de Compostela, Jun. 2012. [Online]. Available: http://www.ac.usc.es/node/1607

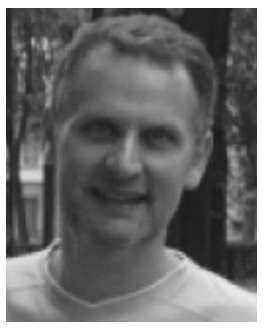

Elisardo Antelo received the graduation degree in physics, in 1991, and the Ph.D. degree in computer engineering, in 1995, from the University of Santiago de Compostela, Santiago de Compostela, Spain.

In 1992, he joined the Departamento de Electronica e Computacion at the University of Santiago de Compostela. From 1992 to 1998, he was an Assistant Professor and, since 1998, he has been a tenured Associate Professor in this department. He was a Research Visitor at the University of California at Irvine several times between 1996 and 2000, and at the Politenico di Torino in 2012. He is a member of the computer architecture group at the University of Santiago de Compostela. Since 2001, he has been involved in the program committee of the IEEE Symposium on Computer Arithmetic (program cochair in the 2011 edition). His primary research and teaching interests include digital design and computer architecture with current emphasis on high-speed and low-power numerical processors, applicationspecific modules, computer arithmetic, and design issues related to multicore processors.

Dr. Antelo was an Associate Editor of the IEEE TRANS ACTIONS ON COMPUTERS (2007-2012), and of the Journal of VLSI Integration (2011-2012).

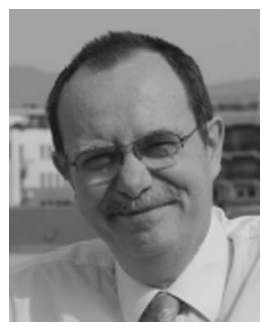

Paolo Montuschi (M'90-SM'07-F'14) received the $\mathrm{Ph} . \mathrm{D}$. degree in computer engineering from Politecnico di Torino, Torino, Italy, in 1989.

Since 2000, he has been a Full Professor of Computer Engineering at Politecnico di Torino, Torino, Italy, where he served as Chair of Department from 2003 to 2011, and as Chair or Member of several Boards including the Board of Governors. His current research interests and scientific achievements are in computer arithmetic, computer graphics, electronic publications, semantics and education, and new frameworks for the dissemination of scientific knowledge.

Dr. Montuschi is serving as Editor-in-Chief of the IEEE TRANSACTIONS ON COMPUTERS, as a member of the steering committee, and as a Member of the Advisory Board of Computing Now. He is also serving as Member-atLarge of the IEEE Publication Services and Products Board and of the IEEE TAB/PSPB Products and Services Committee. Previously, he served as Chair of the Magazine Operations, of the Electronic Products and Services, and of the Digital Library Operations Committees, Member-at-Large of the Computer Societys Publications Board, and Member of the Board of Governors of the IEEE Computer Society. He served as Guest, Associate Editor, and Associate Editor-in-Chief of the IEEE TRANSACTIONS ON COMPUTERS from 2000 to 2004, from 2009 to 2012, and from 2013 to 2014, as well as cochair, program and steering committee member of several conferences. He is a Computer Society Golden Core Member and a Life Member of the International Academy of Sciences of Turin.

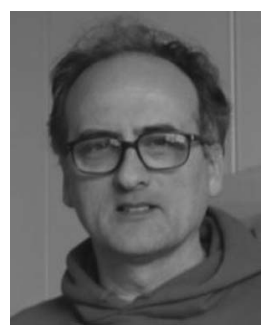

Alberto Nannarelli (S'94-M'99-SM'13) graduated in electrical engineering from the University of Roma "La Sapienza," Roma, Italy, in 1988, and received the M.S. and the Ph.D. degrees in electrical and computer engineering from the University of California at Irvine, CA, USA, in 1995 and 1999, respectively.

He is an Associate Professor at the Technical University of Denmark, Lyngby, Denmark. He worked for SGS-Thomson Microelectronics and for Ericsson Telecom as a Design Engineer and for Rockwell Semiconductor Systems as a summer intern. From 1999 to 2003, he was with the Department of Electical Engineering, University of Roma "Tor Vergata," Italy, as a Postdoctoral Researcher. His research interests include computer arithmetic, computer architecture, and VLSI design.

Dr. Nannarelli is a Senior Member of the IEEE Computer Society. 